\title{
ADAPTIVE PRE- AND POST-FILTERING FOR BLOCK BASED SYSTEMS
}

\author{
Sachin Gangaputra and Trac D. Tran \\ Department of Electrical and Computer Engineering \\ The Johns Hopkins University \\ Baltimore, MD 21218, USA \\ email:\{sachin,trac\}@jhu.edu
}

\begin{abstract}
This paper introduces an adaptive time-varying signal decomposition framework with perfect reconstruction via a combination of adaptive time-domain pre/post-processing and size-adaptive block DCTs. We explore different methods to produce time-varying basis functions and study various properties of the transition filter banks involved. Several criteria that could be used to select a certain set of basis functions are investigated. Promising coding gains over those of non-adaptive decompositions in an image coding setting are also presented.
\end{abstract}

\section{INTRODUCTION}

Most image and video codecs are based on time-invariant transforms. For example, JPEG and MPEG fix the transform to the 8-point DCT. In JPEG2000, the default transform is the dyadic wavelet transform with the 9/7-tap Daubechies filters. It is certainly very advantageous from a rate-distortion viewpoint to be able to adapt the transformation to the local signal characteristics. Two representative research directions in this area are the adaptive wavelet packet decomposition in the wavelet field [1] and DCT decomposition of varying block sizes in the block transform field. These transforms are particular instances of a larger class of filter banks (FBs). [2], [3] characterize and outline properties of such general case time-adaptive filter banks, with regard to image coding applications.

Recently a new signal decomposition framework was introduced that can construct a large family of filter banks by adding simple time-domain pre- and post-processing modules onto existing DCT-based infrastructure [4]. Viewing this framework globally as shown in Fig. 1 reveals its elegance. Choosing $\mathbf{V}$ as

$$
\mathbf{V}=\mathbf{J} \mathbf{C}_{M / 2}^{I I^{T}} \mathbf{S} \mathbf{C}_{M / 2}^{I V} \mathbf{J}
$$

yields very cost-effective solutions for biorthogonal systems. Here the symbols $\mathbf{C}_{M}^{I I}$ and $\mathbf{C}_{M}^{I V}$ denote, respectively, the $M \times M$ typeII and type-IV DCT matrices. $\mathbf{S}$ is chosen as $\operatorname{diag}\{s, 1, \ldots, 1\}$ and $\mathbf{J}$ is the anti-diagonal unity matrix.

Further, changing the size of the pre-processing matrix $\mathbf{P}$ controls the amount of borrowing samples at the particular block boundary, i.e. the length of the local basis functions. This is crucial in applications that need a good trade-off between coding gain and complexity. Thereby this framework lends itself perfectly to

\footnotetext{
This research has been supported by NSF under grant CCR - 0093262
}

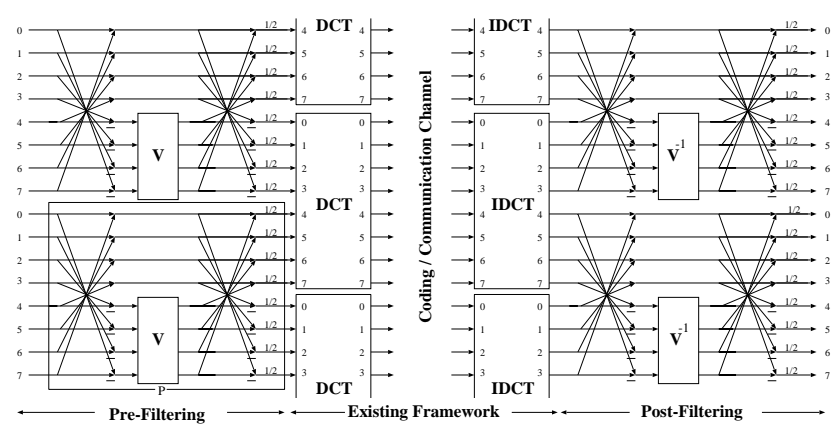

Fig. 1. Signal decomposition and representation with pre- and post-filtering at DCT block boundaries.

the problem of designing adaptive time-varying signal decompositions. In this paper, we inspect different methods to produce adaptive basis functions and examine their perfect reconstruction (PR) conditions based on the aforementioned framework. Further, we introduce an adaptive image coder and compare results of different adaptive strategies with their non-adaptive counterparts. The goal of our system is to modify the number of borrowed samples at each block boundary, or to alter the number of pre/post-processing stages, or to vary the DCT block size-based on the local input signal statistics. Throughout this paper, an $M$-channel, $L$-tap filter bank is denoted as $\mathbf{M} \times \mathbf{L} \mathbf{F B}$.

\section{GENERAL ADAPTIVE DECOMPOSITION}

The signal decomposition framework mentioned above is simply the polyphase implementation of a maximally decimated M-channel filter bank whose polyphase matrix is represented as

$$
\mathbf{E}(z)=\mathbf{C}_{M}^{I I} \hat{\Lambda}(z) \mathbf{P}
$$

where $\hat{\boldsymbol{\Lambda}}(z) \mathbf{P}$ can be interpreted as time-domain pre-processing across block boundaries. Time variation with PR is realizable if the matrix $\mathbf{P}$ remains invertible at all times. Therefore, the matrix $\mathbf{P}$ can be varied along the time axis and can be expressed as $\mathbf{P}(n)$. At time index n, the instantaneous polyphase matrix $\mathbf{E}(z, n)$ can be expressed as

$$
\mathbf{E}(z, n)=\mathbf{C}_{M}^{I I} \hat{\Lambda}(z) \mathbf{P}(n) .
$$

The synthesis polyphase matrix is simply the inverse of $\mathbf{E}(z, n)$.

$$
\mathbf{R}(z, n)=\mathbf{P}^{-1}(n) \hat{\mathbf{\Lambda}}^{-1}(z) \mathbf{C}_{M}^{I I^{T}}
$$



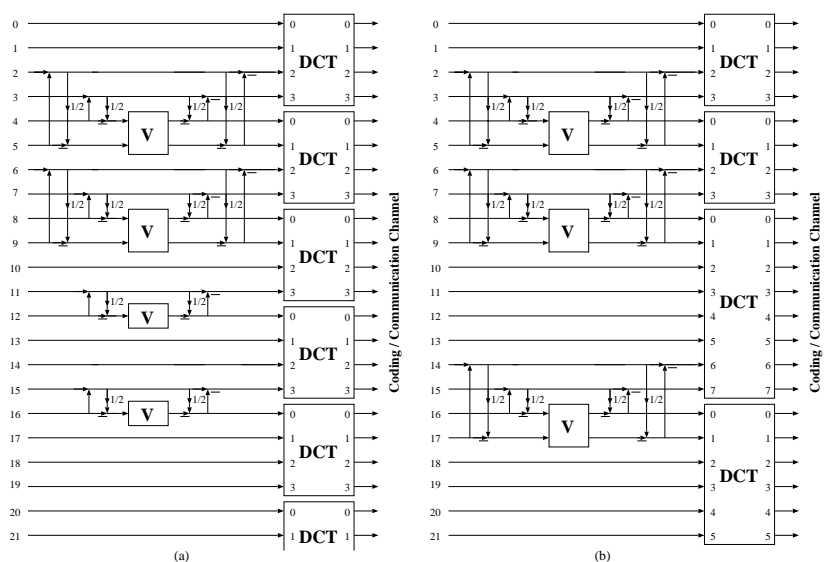

Fig. 2. Adaptive time-varying signal decomposition. (a) Adaptive borrowing, fixed DCT block size. (b) Adaptive DCT block size, fixed borrowing.

\section{A. Varying number of borrowing.}

This scheme involves no restrictions on the amount of samples we wish to borrow from neighboring blocks. Unequal numbers of samples borrowed from each side of a block yields an instantaneous transitional filter bank with non-linear-phase basis functions. We are more interested in maintaining PR than in maintaining linear phase since the number of transitional FBs should be rather limited. The adaptive-borrowing scheme is demonstrated in Fig. 2(a) where the block size is fixed at 4 while the pre-filtering operation can be chosen amongst: no filtering, borrowing 1 sample, or borrowing 2 samples. Here we are switching from a $4 \times 8$ to a $4 \times 7$ to a $4 \times 6$ to a $4 \times 5$ FB and possibly to a 4 -point DCT. The polyphase matrix of this scheme is as given in (3). PR is guaranteed as all $\mathbf{P}$ matrices are chosen to be invertible. In general, this scheme yields an $\mathbf{M} \times\left(\mathbf{M}+\mathbf{N}_{a}+\mathbf{N}_{b}\right) \mathrm{FB}$, where $\mathbf{N}_{a}$ and $\mathbf{N}_{b}$ denote the number of samples borrowed from the block above and the block below respectively.

\section{B. Varying block size.}

Adaptivity can be obtained by employing variable block sizes. We would prefer to have larger blocks for slower signal transitions and smaller blocks for fast changing transient parts of the signal. Such signal adaptive schemes have been used effectively in practice by the MPEG-4 audio coder. The variable block size scheme is illustrated in Fig. 2(b). Here, we are switching from a $4 \times 8$ to an $8 \times 12$ to a $6 \times 10 \mathrm{FB}$. The polyphase matrix can be represented as

$$
\mathbf{E}(z, n)=\mathbf{C}_{M(n)}^{I I} \hat{\Lambda}(z) \mathbf{P} .
$$

Again, PR is guaranteed by the invertibility of $\mathbf{C}_{M(n)}^{I I}$ and $\mathbf{P}$.

\section{Varying number of stages.}

Alternately, adding different numbers of stages of pre- and post-processing adaptively results in another adaptive decomposition scheme. Each added pre-processing stage $\mathbf{P}_{i}$ works at the boundaries of the previous $\mathbf{P}_{i-1}$. The instantaneous filter bank generated is an $\mathbf{M} \times(\mathbf{K}(n) \mathbf{M}+\mathbf{N}) \mathbf{F B}$, where $\mathbf{K}(n)$ is the number of pre-processing stages at time $n$ and $\mathbf{N}$ is the total number of samples borrowed in each pre-processing stage. This scheme is illustrated in Fig. 3.
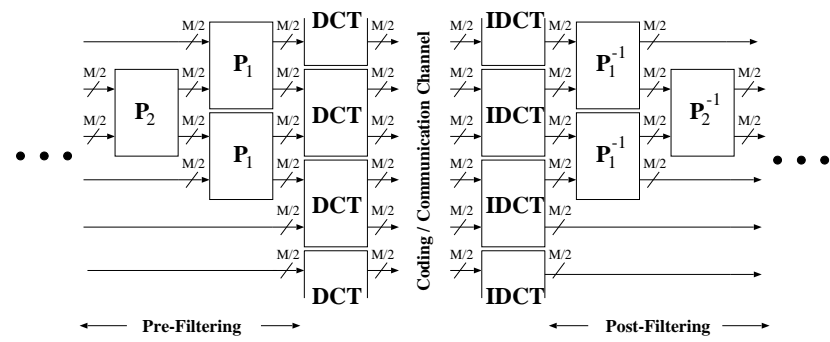

Fig. 3. Pre- and post-filtering with adaptive number of stages

The analysis polyphase matrix can be constructed modularly as

$$
\mathbf{E}(z, n)=\mathbf{C}_{M}^{I I} \prod_{i=1}^{K(n)}\left[\hat{\boldsymbol{\Lambda}}(z) \mathbf{P}_{i}(n)\right]
$$

PR is guaranteed by choosing all $\mathbf{P}_{i}(n)$ to be invertible.

A generalized adaptive scheme can be designed as a combination of the above-mentioned methods. The polyphase of this general framework will then take the form

$$
\mathbf{E}(z, n)=\mathbf{C}_{M(n)}^{I I} \prod_{i=1}^{K(n)}\left[\hat{\boldsymbol{\Lambda}}(z) \mathbf{P}_{i}(n)\right]
$$

In the remaining sections, the adaptive-borrowing case will be our main focus. We shall study the corresponding FB characteristics and evaluate coding results.

\section{TRANSITION FILTERS}

Transition filters are time-varying filters used in changing from one type of time-invariant filter bank to another. A lot of research has been done on the design of optimal transition filters [5], [6] and this section briefly describes the characteristics of the transition filters used in our framework.

As shown in Fig. 2(a), we encounter the transition $4 \times 7 \mathrm{FB}$ when moving from a $4 \times 8$ to a $4 \times 6 \mathrm{FB}$. A transition within a single stage of preprocessing affects two neighboring blocks. That is, for a transition in the $\mathbf{K}^{t h}$ stage a total of $\mathbf{K}+1$ blocks are involved. Table 1 shows the transition coding gains with various amounts of borrowing from the blocks above and below. Note that borrowing $N_{a}$ samples from the block above and $N_{b}$ samples from the block below or borrowing $N_{b}$ samples from the block above and $N_{a}$ samples from the block below gives us equivalent (up to a timeflip) transition filters. These coding gains are encouraging and lead us to design a practical image coder. The analysis/synthesis time and frequency responses for two cases of transition are shown in Fig. 4. As can be seen from the time and frequency responses of the filters involved, there is a nonlinear phase. Linear phase can only be guaranteed for the adaptive block size scheme outlined in section 2-B as there we have equal borrowing from both sides of the involved block and we can use

$$
\mathbf{N} \leq \min \left(\mathbf{M}_{i}\right) / 2
$$

where $\mathbf{N}$ is the total number of borrowed samples from each side of a block and $\mathbf{M}_{i}$ is the set of permissible block sizes. 

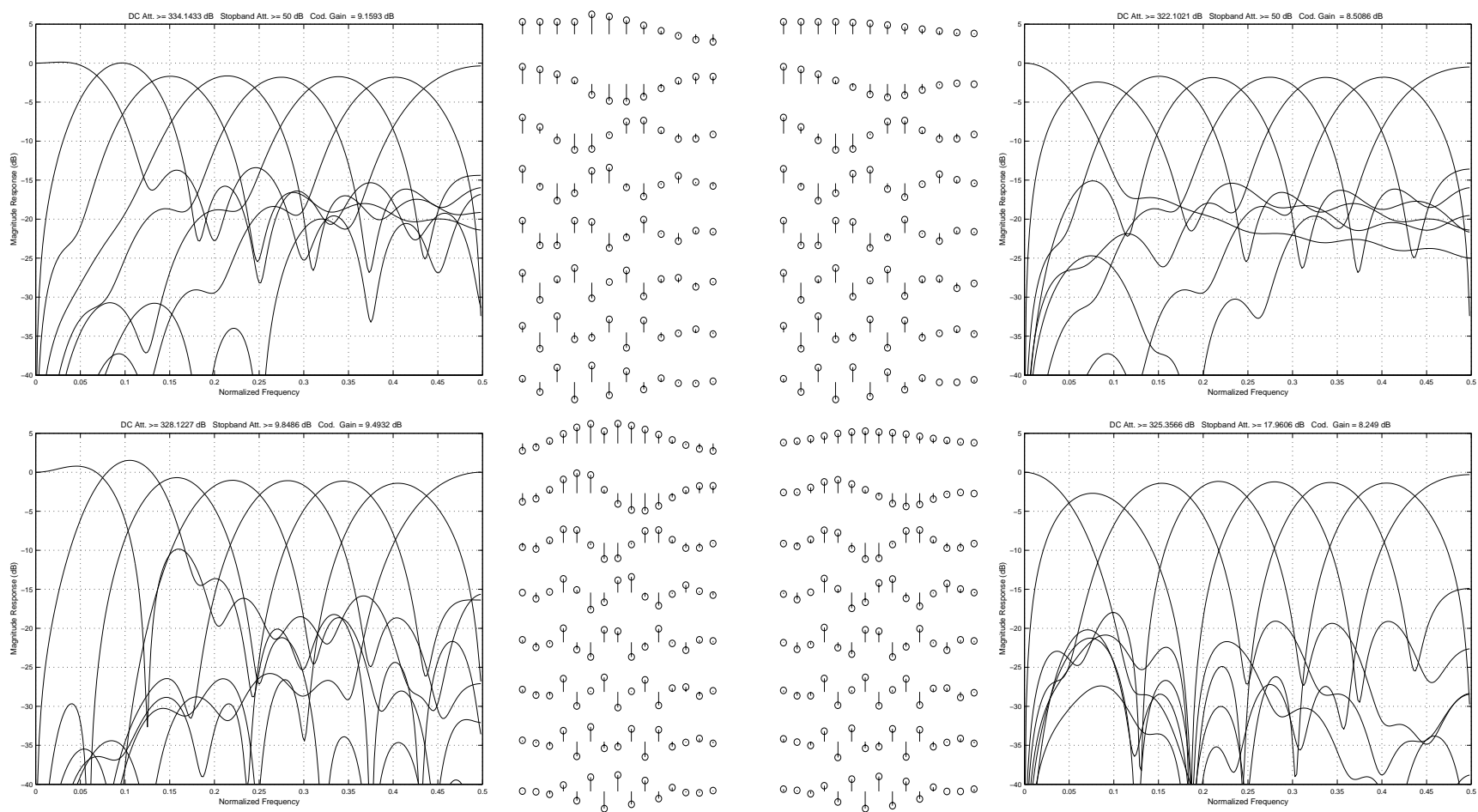

Fig. 4. From left to right: analysis frequency responses, analysis time responses, synthesis time responses, and synthesis frequency responses. From top to bottom: $8 \times 12$ transition FB via no filtering above and 4-point pre/post-filtering below; $8 \times 15$ transition FB via 3 -point pre/post-filtering above and 4-point pre/post-filtering below.

Table 1. Coding gains for various transition FBs

\begin{tabular}{|c|c|c|}
\hline$N_{a}$ & $N_{b}$ & Coding gain \\
\hline 0 & 0 & 8.83 \\
\hline 0 & 1 & 8.94 \\
\hline 0 & 2 & 9.05 \\
\hline 0 & 3 & 9.12 \\
\hline 0 & 4 & 9.16 \\
\hline 1 & 1 & 9.06 \\
\hline 1 & 2 & 9.18 \\
\hline 1 & 3 & 9.24 \\
\hline 1 & 4 & 9.28 \\
\hline 2 & 2 & 9.05 \\
\hline 2 & 3 & 9.37 \\
\hline 2 & 4 & 9.41 \\
\hline 3 & 3 & 9.45 \\
\hline 3 & 4 & 9.49 \\
\hline 4 & 4 & 9.55 \\
\hline
\end{tabular}

\section{ADAPTIVE IMAGE CODER}

An adaptive image codec based on JPEG is implemented to verify the performance of the proposed adaptive pre/post-filtering scheme. Here the transform is fixed to be an 8-point DCT and we add adaptive pre- and post-processing modules between each block boundary. The free-parameter matrix $\mathbf{V}$ is always chosen as in (1); however, its size varies depending on several different criteria discussed below.
MSE BASED: This criterion is based on the intuition that the lower the energy of the signal fed to the DCT; the higher would be its compression ratio.

OVER/UndeRFLOW BASED: Pre-processing can increase the dynamic range of the input signal samples fed to the DCT. This criterion regards coefficients above/below the conventional DCT range [0-255] as inapt and chooses an alternate filter bank. Experiments reveal that over/underflow occurs at edges and a simple edge detector would suffice as a good alternative.

NUMBER OF DCT ZERO COEFFICIENTS: This is a more robust scheme that computes the DCT of the pre-processed samples, quantizes them and chooses the pre-filter that yields the maximum number of zero coefficients. This is based on the intuition that the greater the number of quantized zeros, the more efficient the following entropy coding algorithm.
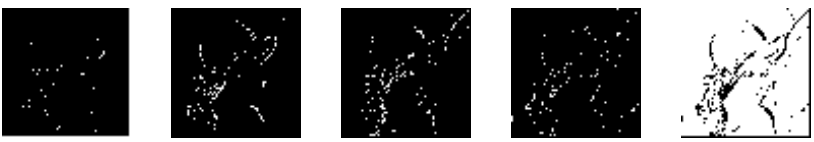

Fig. 5. Block selection for Lena (35:1): White indicates selected block. From left to right: 8 -point DCT, $8 \times 10 \mathrm{FB}, 8 \times 12 \mathrm{FB}$, $8 \times 14 \mathrm{FB}, 8 \times 16 \mathrm{FB}$.

Our implementation is based on the conventional separable method used in image coders. Optimal filter banks are sought progressively by traversing the blocks in a left-right, top-down man- 

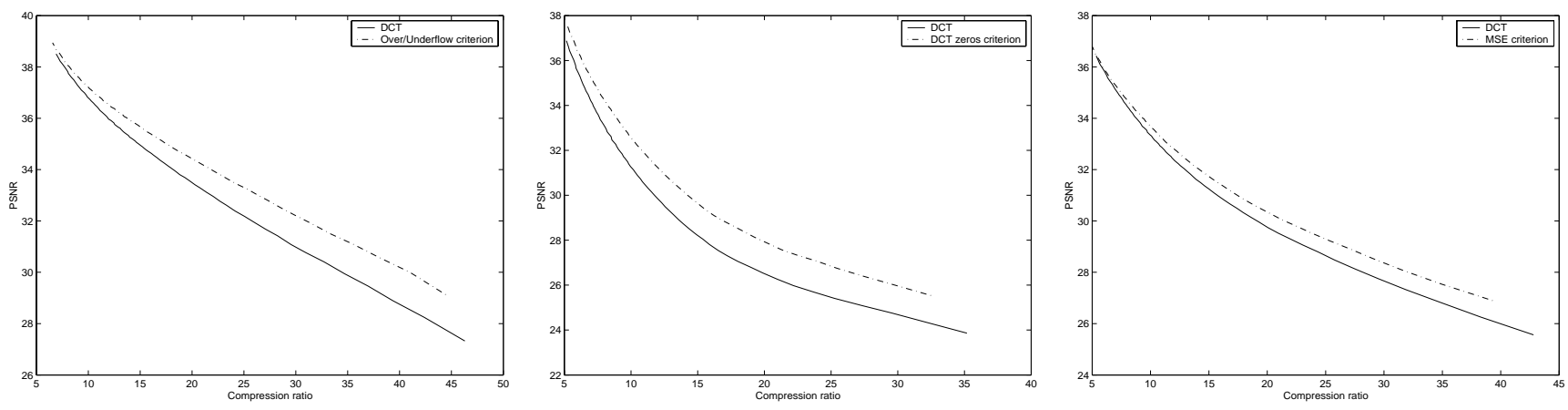

Fig. 6. PSNR Curves: From left to right: Lena, Barbara and Boat using Over/Underflow, DCT zeros and MSE criterion respectively.

ner. Each block is tested with the permissible set of filter banks and the filter bank satisfying the predetermined criterion is selected for pre-processing. If we have more than one filter bank satisfying the criterion we select the filter bank with the least borrowing, leading to a less complex encoder. Fig. 5 shows masks denoting the different selected filters for the different $8 \times 8$ blocks of Lena. Here, we have implemented the over/underflow criterion. From left to right of the figure the corresponding blocks choose 0,1,2,3,4-sample borrowing from each side. As expected we see a maximum sample borrowing in smoother regions and no borrowing along edges. Other criteria yield similar block selection masks.

The PSNR curves for these schemes in comparison to nonadaptive image coding results are plotted in Fig. 6. Each criterion gives us similar curves with an average PSNR improvement of 1.4 $\mathrm{dB}$. The pre-processed samples are truncated to fit the 8-bit range, making them compliant with the baseline JPEG encoder. Despite this disadvantage we see tremendous coding gain. One drawback of the adaptive scheme is the overhead generated in communicating the instantaneous filter bank being used to the decoder. As the instantaneous filter bank is chosen from a well defined set, known both to the decoder and encoder, it would suffice to encode a corresponding index of the selected filter bank. The resulting overhead can be kept manageable with a well-chosen set of filter banks and by applying some run-length coding on the resulting set of chosen indices. The overhead for our test images was approximately 0.012 bpp.

\section{CONCLUSION}

We have presented a general PR signal decomposition framework based on adaptive time-varying pre- and post-filtering for block DCT-based systems. The framework has the advantage of being a modular structure that allows FBs of varying-length filters while maintaining a fast, efficient, and elegant block-based implementation. We also demonstrate the proposed adaptive framework's effectiveness in a simple image coding experiment.

\section{REFERENCES}

[1] M. V. Wickerhauser, 'Adapted Waveform Analysis, WaveletPackets and Applications', SIAM Press, 1992.

[2] S.-M. Phoong and P.P. Vaidyanathan, 'Time-Varying Filters and Filter Banks: Some basic principles', IEEE trans. Signal Processing, vol. 44, pp. 2971-2987, Dec 1996.
[3] S.-M. Phoong and P.P. Vaidyanathan, 'A polyphase approach to time-varying filter banks', ICASSP'96, Atlanta, GA, May 1996. pp. $1554-1557$ vol. 3.

[4] Trac D. Tran, 'Lapped Transform via Time-Domain Pre- and Post-Processing', Conference on Information Science and Systems,pp. 890-895 March 21, 2001.

[5] C. Herley, 'Boundary filters for finite-length signals and timevarying filter banks', IEEE Trans. Circuits and Systems II, vol. 42, Feb. 1995.

[6] T. Kalker, 'On optimal boundary and transition filters in time varying filter banks', ICIP'96, Lausanne, Switzerland, September 1996. Page(s): 625 -628 vol.1.

[7] R. de Querioz and K.R. Rao, 'Time-varying lapped transform and wavelet packets', IEEE transactions on signal processing, vol. 41, pp. 3293-3305, Dec 1993.

[8] R. de Querioz and K.R. Rao, 'Adaptive extended lapped transforms', ICASSP'93. Minneapolis, April 1993. Page(s): 217 -220 vol.3.

[9] T.J. Klausutis and V.K. Madisetti, 'Adaptive lapped transform based image coding', IEEE Signal Processing Letters, vol: 4. pp: $245-247$, Sept. 1997. 